\title{
Dynamics and freeze-out of hadron resonances at RHIC
}

\author{
Marcus Bleicher, Horst Stöcker \\ Institut für Theoretische Physik, J. W. Goethe Universität, 60054 Frankfurt am \\ Main, Germany
}

\begin{abstract}
Yields, rapidity and transverse momentum spectra of $\Delta^{++}(1232)$, $\Lambda(1520), \Sigma^{ \pm}(1385)$ and the meson resonances $K^{0}(892), \Phi, \rho^{0}$ and $f_{0}(980)$ are predicted. Hadronic rescattering leads to a suppression of reconstructable resonances, especially at low $p_{\perp}$. A mass shift of the $\rho$ of $10 \mathrm{MeV}$ is obtained from the microscopic simulation, due to late stage $\rho$ formation in the cooling pion gas.
\end{abstract}


Strange particle yields and spectra are key probes to study excited nuclear matter and to detect the transition of (confined) hadronic matter to quark-gluon-matter (QGP) [1, 2]. The relative enhancement of strange and multi-strange hadrons, as well as hadron ratios in central heavy ion collisions with respect to peripheral or proton induced interactions have been suggested as a signature for the transient existence of a QGP-phase [2]. A main difficulty in the interpretation of the available data is that the observed final state hadrons carry relatively little information about their primordial sources. Most of the hadrons had been subject to many secondary interactions and are strongly influenced by the decays of high mass resonances.

Recently, first direct experimental information on unstable particle emission in nucleus-nucleus reactions has been reported. At SPS [3], the $\Phi, \overline{K^{0}}(892)$ and $\Lambda(1520)$ have been observed, at the full RHIC energy first data on the $\Phi, K^{0}(892), f_{0}$ and $\rho$ mesons and the $\Lambda(1520)$ and $\Sigma(1385)$ baryon resonances are available [4]. These data are obtained by the reconstruction of final state hadrons. Thus, in contrast to the study of $\rho$ mesons by di-leptons (which are penetrating probes), it yields information mostly from the later stages of the reaction. Therefore, the question of the existence of such resonance states in the hot and dense environment is still not unambiguously answered. E.g. hyperon resonances are expected to dissolve at high energy densities [6] and also the $\rho$ is expected to "melt" in the medium [5]. However, especially for the strange baryon resonances it is expected that late stage regeneration of the resonance is suppressed, therefore there yield might still carry information whether they do exist in the hot and dense region. It is therefore of utmost importance to study the cross section of hyperon resonance production at different beam energies and centralities to see whether a specific suppression threshold is present.

To study a possible suppression of resonances and its origin, the Ultra-relativistic Quantum Molecular Dynamics model (UrQMD 1.2) is used [7. This microscopic transport approach is based on the covariant propagation of constituent quarks and diquarks accompanied by mesonic and baryonic degrees of freedom. The leading hadrons of the fragmenting strings contain the valence-quarks of the original excited hadron and represent a simplified picture of the leading (di)quarks of the fragmenting string. The elementary hadronic interactions are modelled according to measured cross sections and angular distributions. If the cross sections are not experimentally known, detailed balance is employed in the energy range of resonances. The partial and total decay widths are taken from the Particle Data Group. Presently, in-medium modifications of the particle properties, i.e. temperature and density dependent masses and decay widths, are not included in the model. Thus, it serves as a benchmark calculation and allows in comparison to experimental data to identify a possible anomalous behaviour in the resonance dynamics. In these proceedings, we will focus on nucleus-nucleus collisions at RHIC energies. For a discussion of resonance production at SPS and resonance excitation functions within the same approach the reader is referred to Refs. [8, 9]. An exploration of the freeze-out conditions in heavy ion reactions from the perspective of a refined thermal model can be found in [10, 11, 12]. 

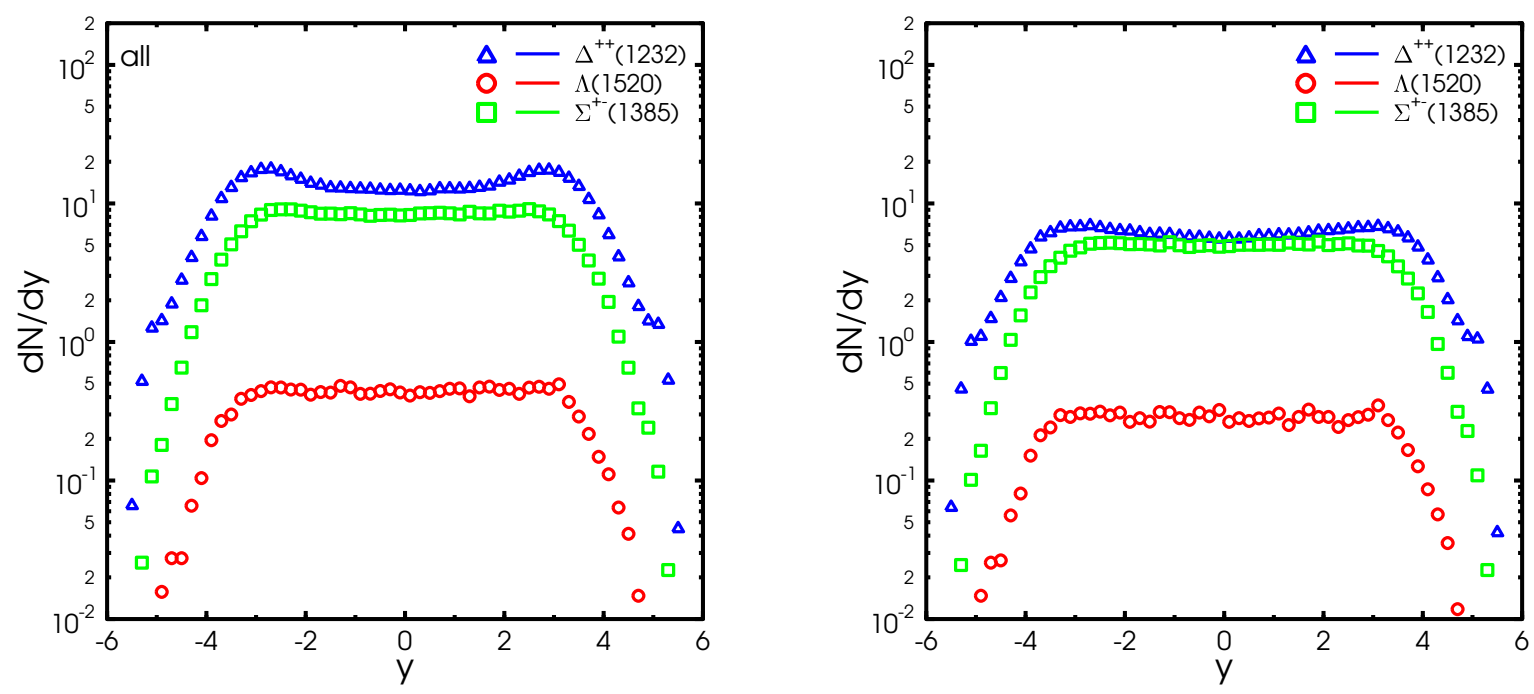

Figure 1. Rapidity densities of baryon resonances in central $\mathrm{Au}+\mathrm{Au}$ reactions at $\sqrt{s}=200 \mathrm{AGeV}$. Left: All baryons resonances that decay are shown. Right: Only those baryon resonances are shown that can (in principle) be reconstructed by experiment because their decay products did not interact after the decay of the resonance.
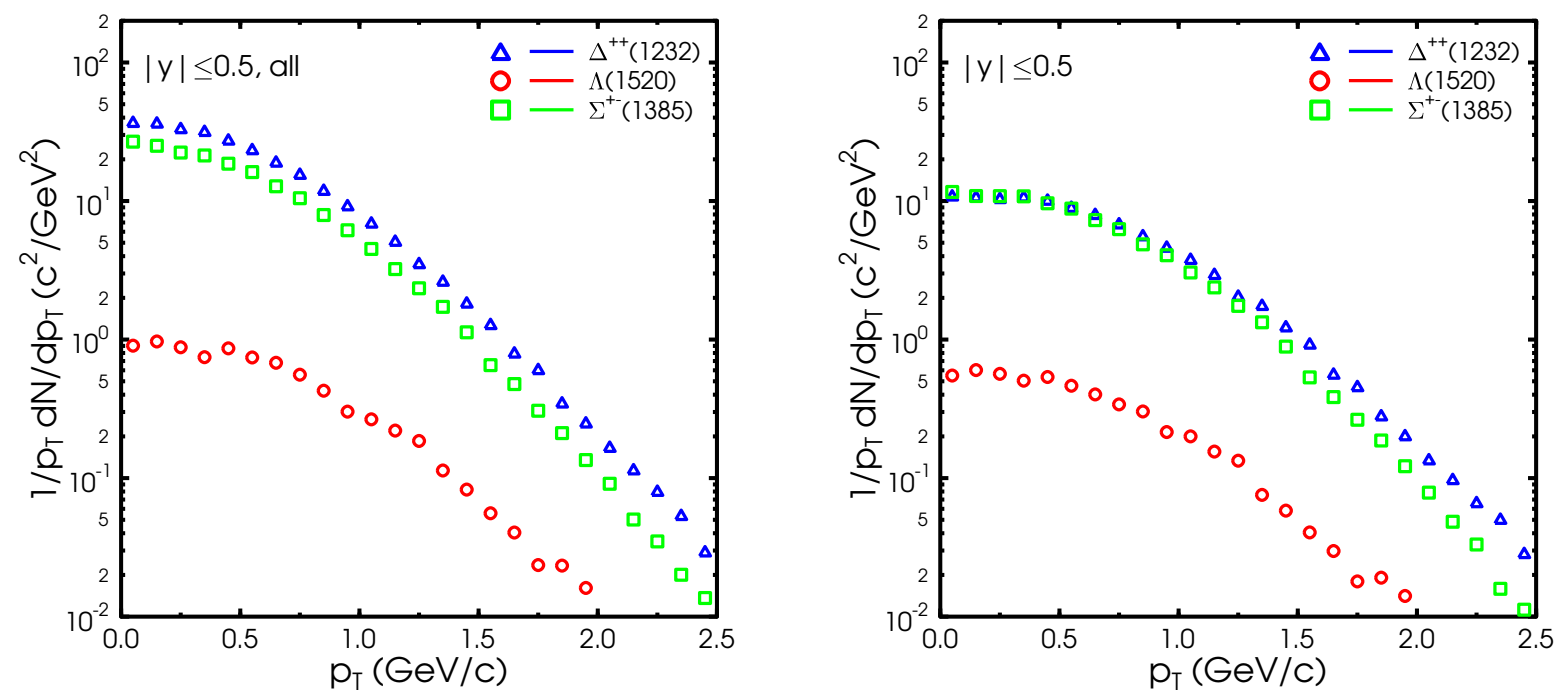

Figure 2. Transverse momentum spectra of baryon resonances in central $\mathrm{Au}+\mathrm{Au}$ reactions at $\sqrt{s}=200 \mathrm{AGeV}$. Left: All baryons resonances that decay are shown. Right: Only those baryon resonances are shown that can (in principle) be reconstructed by experiment. 

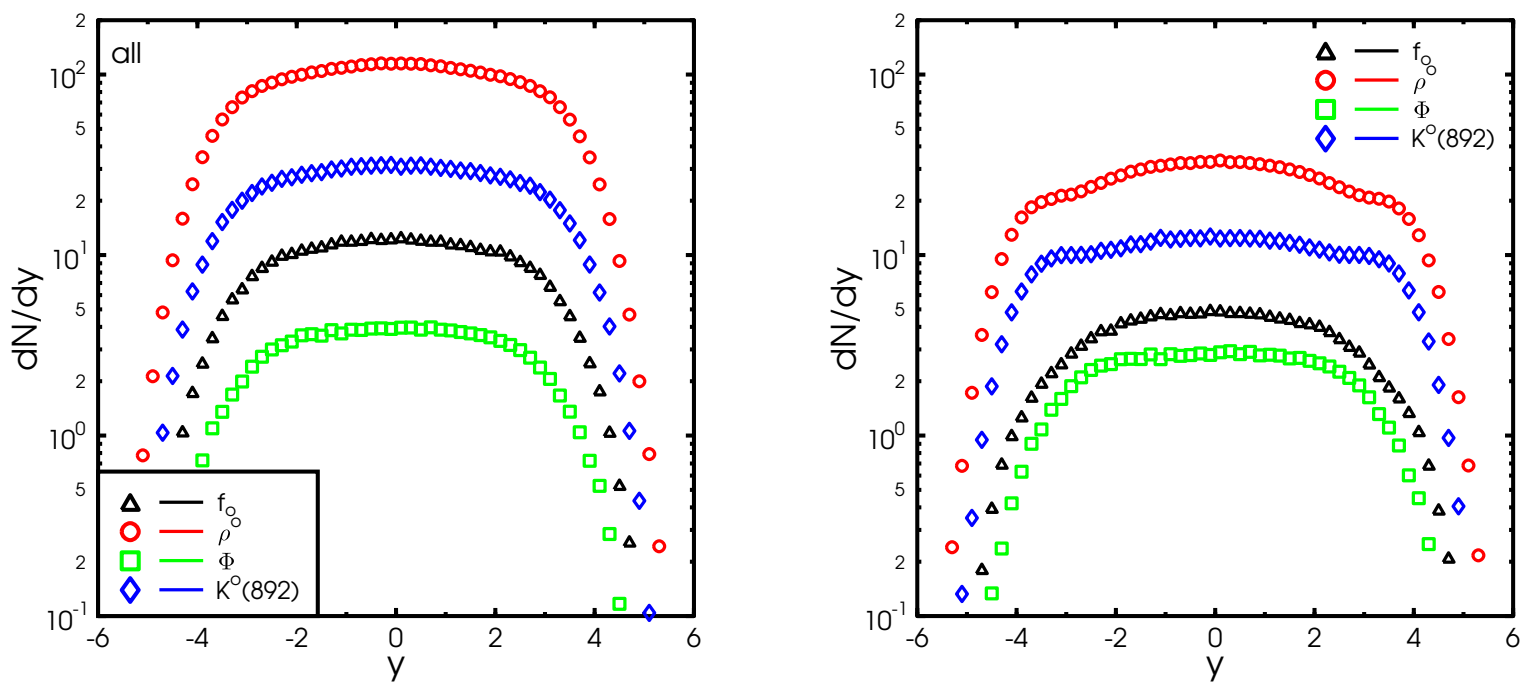

Figure 3. Rapidity densities of meson resonances in central $\mathrm{Au}+\mathrm{Au}$ reactions at $\sqrt{s}=200 \mathrm{AGeV}$. Left: All meson resonances that decay are shown. Right: Only those meson resonances are shown that can (in principle) be reconstructed by experiment because their decay products did not interact after the decay of the resonance.
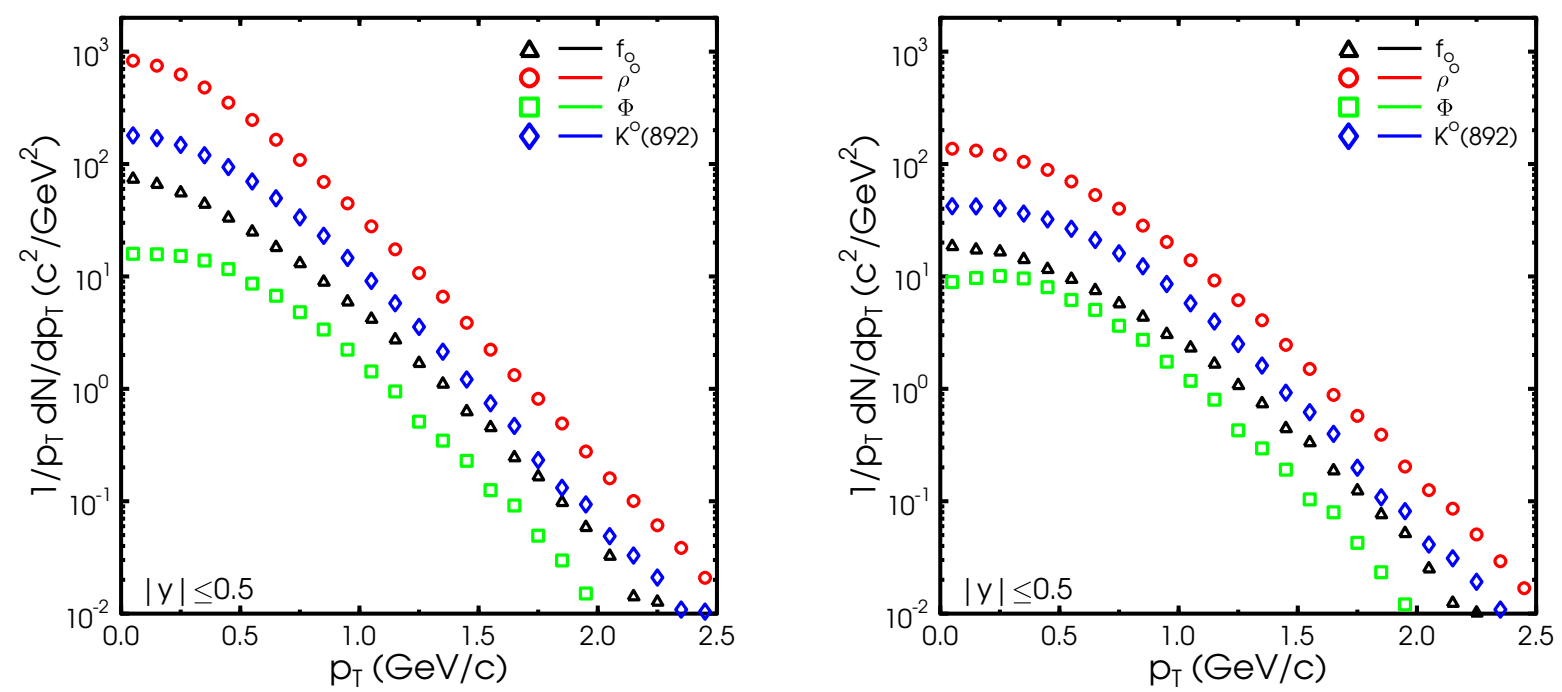

Figure 4. Transverse momentum spectra of meson resonances in central $\mathrm{Au}+\mathrm{Au}$ reactions at $\sqrt{s}=200 \mathrm{AGeV}$. Left: All meson resonances that decay are shown. Right: Only those meson resonances are shown that can (in principle) be reconstructed by experiment. 
In general, a nucleus-nucleus collision evolves through three distinct stages: (I) The initial stage is characterised by high scattering rates and deposits a large amount of (non-thermalized) energy around central rapidities. (II) Either partonic or hadronic "cooking" may equilibrate the system until the individual inelastic scattering rates drop as the system cools down - usually called the chemical freeze-out. (III) Elastic and pseudo-elastic interactions (e.g. $\pi \pi \rightarrow \rho \rightarrow \pi \pi$ ) dominate the final stage of the collision, ending with the final break-up of the "fireball" (kinetic freeze-out). The spectra and abundances of meson and baryon resonances are well suited to study the break-up dynamics of the source between chemical and kinetic freeze-out.

Let us start by an overview of the rapidity and transverse momentum spectra in central $\mathrm{Au}+\mathrm{Au}$ collisions at $\sqrt{s}=200 \mathrm{AGeV}$. Fig. 1 shows the rapidity densities of the $\Delta^{++}(1232), \Lambda(1520)$ and the charged $\Sigma^{ \pm}(1385)$ states in central Au+Au reactions at $\sqrt{s}=200 \mathrm{AGeV}$. The left part of Fig. 1] includes all decaying baryon resonances in the spectrum, while the right part of Fig. 1 shows the finally observable spectrum of resonances. Different to the experiment, were a mixed event technique is used to obtain an invariant mass spectrum at any given $p_{\perp}$ and rapidity, in the present study we define a resonance as observable if its decay products do not interact further after the decay of the resonance. This procedure has the advantage that for each individual observed resonance information on its origin and decay time can be obtained from the model. However, this definition might yield a slightly stronger suppression of resonances than the mixed event technique, because it assumes that any momentum transfer to the decay products will result in a complete loss of the resonance signal in the invariant mass spectrum of the two correlated hadrons.

Fig. 2 shows the transverse momentum spectra of $\Delta^{++}(1232), \Lambda(1520)$ and the charged $\Sigma^{ \pm}(1385)$ states in central $\mathrm{Au}+\mathrm{Au}$ reactions at $\sqrt{s}=200 \mathrm{AGeV}$. Here, one observes a significant reduction of the low $p_{\perp}$ part of the spectrum, while the high $p_{\perp}$ part remains essentially unchanged. This apparent heating of the resonance spectrum is partly due to flow, but mostly due to the higher absorption probability for the decay products of low $p_{\perp}$ resonances because they decay already inside the hot and dense region.

Figures 3 and 4 show the same information for the meson resonances $K^{0}(892), \Phi$, $\rho^{0}$ and the neutral $f_{0}(980)$. The left parts of the figures include all resonances as they decay, while the right parts show only those resonances that are reconstructable from an invariant mass spectrum of final state hadrons. I.e. the left parts of Figs. 3 and 4 can be studied in the di-lepton channel (e.g. in the $\rho^{0}$ decay, normalised by the branching ratio $\Gamma_{\rho \rightarrow l^{+} l^{-}}$), while the right parts show predictions for the spectra by reconstruction of $K \pi, \bar{K} K$ and $\pi \pi$ hadron correlations.

Especially the $\rho$ meson with its two distinct decay modes $\left(e^{+} e^{-}\right.$vs. $\left.\pi \pi\right)$, which are both accessible by experiment, allows to explore the dynamics and thermodynamic properties of the system. Therefore, we will now focus on the freeze-out and decay of $\rho$ mesons.

In Figure 5 (left) the transverse radii at which $\rho^{0}$ mesons decay in central $\mathrm{Au}+\mathrm{Au}$ 

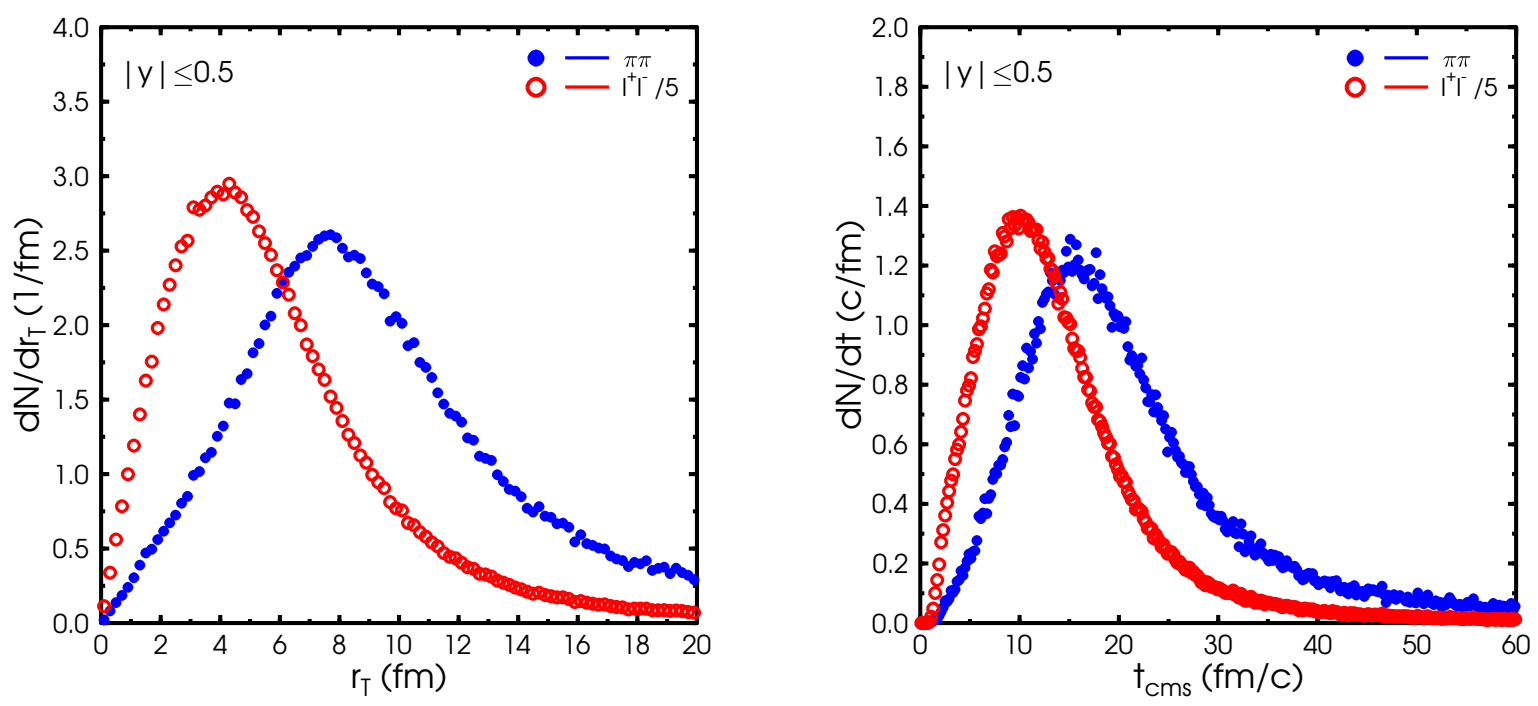

Figure 5. Time and transverse position of the $\rho$ decay around midrapidity $(|y| \leq 0.5)$ in central $\mathrm{Au}+\mathrm{Au}$ reactions at $\sqrt{s}=200 \mathrm{AGeV}$. Closed symbols denote decaying $\rho$ mesons reconstructable in the pion channel, open symbols show the di-lepton channel (normalised by the branching ratio). Left: Transverse radii at which the $\rho^{0}$ mesons decay Right: Center of mass times at which the $\rho^{0}$ mesons decay.

reactions at the full RHIC energy are shown. Open symbols depict the di-lepton channel (divided by 5 for better visibility and normalised by the respective branching ratio), full symbols show the $\rho^{0}$ 's reconstructable in the pion channel. One clearly observes that the $\pi \pi$ channel is mainly sensitive to the outer layers of the interaction region. Here, the emission peaks at $r_{\perp}=8 \mathrm{fm}$. Studying the $\rho$ in the di-lepton channel however, reveals information mainly from the interior of the reaction zone, with a peak around $r_{\perp}=4 \mathrm{fm}$.

The same behaviour is reflected in the decay times of the $\rho$ 's at midrapidity (see Fig. 5 (right)). While the pion channel automatically triggers on the final stages of the interaction (full symbols) with peak emission at $t=15 \mathrm{fm} / \mathrm{c}$, the di-lepton channel, shown as open symbols, probes the density and temperature evolution around $10 \mathrm{fm} / \mathrm{c}$ after the begin of the interaction.

It is interesting to note that the $\rho$ yield as observed in the pion channel is dominated by regenerated $\rho$ 's. I.e. since the life time of the $\rho$ state is $\sim 1.5 \mathrm{fm} / \mathrm{c}$, the finally observed $\rho$ mass spectrum and yield is sensitive to the temperature and densities of the pions near the final break-up of the system.

Indeed, preliminary STAR data [13] seems to indicate a mass shift in the $\rho$ if reconstructed by pion correlations. A first rough estimate of the $\rho$ mass shift due to a cooling down of the pion gas can be obtained by folding the Breit-Wigner distribution 
of the $\rho$ mass with a thermal distribution of temperature $T$ :

$$
\frac{\mathrm{d} N_{\rho}}{\mathrm{d}^{3} p \mathrm{~d} m} \sim \exp \left[-E_{\rho} / T\right] A(m) \quad ; \quad A(m) \sim \frac{\Gamma_{\rho}}{\left(m-m_{\rho}^{\text {peak }}\right)^{2}+\left(\Gamma_{\rho} / 2\right)^{2}} \quad,
$$

for simplicity, phase space factors that modify the tails of the mass distribution are not included here. Thus, the finite width of the $\rho$ results in a temperature dependence of the peak mass of the $\rho$ as given by

$$
\frac{\mathrm{d} N_{\rho}}{\mathrm{d} m} \sim m^{2} T K_{2}(m / T) A(m)
$$

For decoupling temperatures of interest, $100 \mathrm{MeV} \leq T \leq 150 \mathrm{MeV}$, this leads to a downward mass shift of the $\rho$ peak by about $10-20 \mathrm{MeV}$. Note that in contrast to this simple estimate, the model calculations always includes phase space corrections and mass dependent decay widths for resonances.

Let us compare these estimates for the $\rho$ mass shift to the results obtained in the present model. In Figure 6 the centrality dependence of the $\rho$ mass spectrum is shown for minimum biased $\mathrm{Au}+\mathrm{Au}$ reaction at RHIC. When going from peripheral to central collisions one finds a small mass shift in the $\rho$ distribution on the order of $10 \mathrm{MeV}$. This indicates, that the increased rescattering in central collisions and the regeneration of the $\rho$ due to pion rescattering in the late stage of the reaction can indeed lead to a shift in the $\rho$ mass.

To explore this behaviour further, Fig. 7 shows the mass distribution of the $\rho^{0}$ mesons being reconstructed in the $\pi \pi$ channel in central $\mathrm{Au}+\mathrm{Au}$ collision at $\sqrt{s}=200 \mathrm{AGeV}$ for different $p_{\perp}$ windows. Here, the present model predicts a strong transverse momentum dependence of the $\rho$ mass shift. At low $p_{\perp}$ the mass shift exceeds $30 \mathrm{MeV}$, while it vanishes above $1.5 \mathrm{GeV}$ in transverse momentum. This observation also supports, the interpretation of the $\rho$ mass shift in the model as a signal for late stage formation of $\rho$ 's due to $\pi \pi$ scattering.

Comparing to the preliminary STAR data [13] which yields downward shift of the $\rho$ mass on the order of $60-70 \mathrm{MeV}$, the observed mass shift in the simulation is too small. It has been suggested that the additional mass shift can be interpreted as the onset of Brown-Rho scaling and various other in-medium modifications of the $\rho$ not included in the present calculation [14, 15].

In conclusion, a microscopic transport approach is used to study hadron resonance production in $\mathrm{Au}+\mathrm{Au}$ reactions at $\sqrt{s}=200 \mathrm{AGeV}$. Rapidity and transverse momentum spectra of reconstructable (strange) meson and baryon resonances are predicted. The effects of hadronic rescattering on the resonance yields and spectra are discussed. Special emphasise is put on the $\rho$ mesons, here a comparison between the di-lepton channel and the $\pi \pi$ channel might allow to measure the length of the kinetic rescattering stage in nucleus-nucleus collisions: the di-lepton channel is sensitive to the intermediate stage of the reaction, while the $\pi \pi$ channel yields information about the kinetic freeze-out stage close to the break-up of the hadronic system. The hadron dynamics near the break-up leads to a mass reduction of the $\rho$ by $10-20 \mathrm{MeV}$ in central reactions most pronounced at low $p_{\perp}$. The mass reduction vanishes towards peripheral reactions and high $p_{\perp}$. 


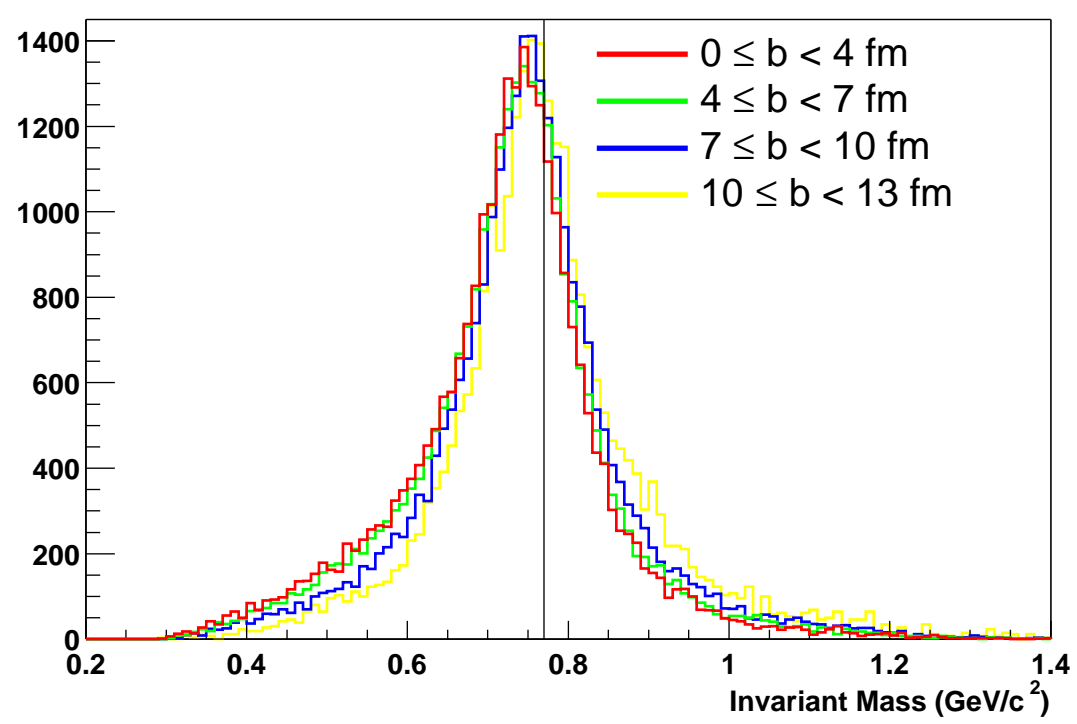

Figure 6. Centrality dependence of the mass distribution of $\rho^{0}$ mesons being reconstructed in the $\pi \pi$ channel in minimum biased $\mathrm{Au}+\mathrm{Au}$ reactions at $\sqrt{s}=$ $200 \mathrm{AGeV}$.

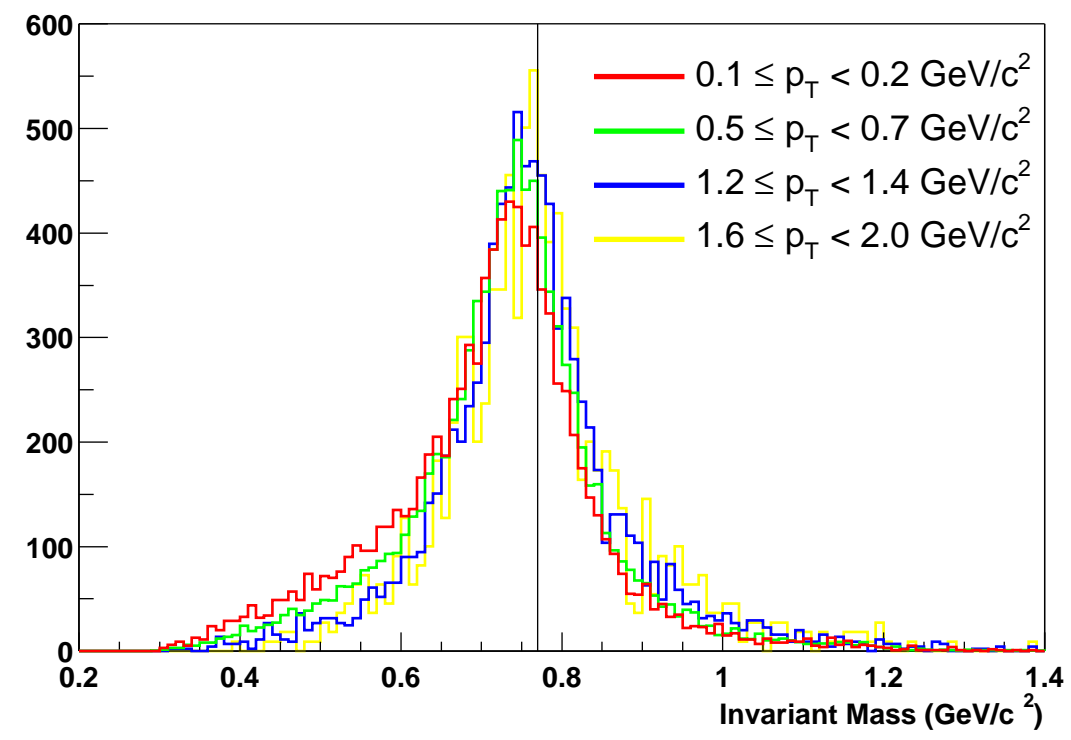

Figure 7. Transverse momentum dependence of the mass distribution of $\rho^{0}$ mesons being reconstructed in the $\pi \pi$ channel in central $\mathrm{Au}+\mathrm{Au}$ reactions at $\sqrt{s}=200 \mathrm{AGeV}$. 


\section{Acknowledgements}

M.B. wants to thank the STAR group at BNL and the Yale University for kind hospitality while working on parts of this manuscript. Fruitful discussion with Patricia Fachini, Zhangbu Xu, Christina Markert, Jörg Aichelin and Edward Shuryak are

gratefully acknowledged. This work used computational resources provided by NERSC and the Center for Scientific Computing at Frankfurt (CSC).

\section{References}

[1] S. A. Bass, M. Gyulassy, H. Stöcker and W. Greiner, J. Phys. G25, R1 (1999); Stock R, Phys. Lett. B456, (1999) 277

[2] J. Rafelski and B. Müller, Phys. Rev. Lett. 48, 1066 (1982);

J. Rafelski, Phys. Rep. 88, 331 (1982);

P. Koch, B. Müller and J. Rafelski, Phys. Rep. 142, 167 (1986).

[3] Ch. Markert, PhD thesis, Univ. Frankfurt; V. Friese [NA49 Collaboration], http://www.rhic.bnl.gov/qm2001/

[4] Z. Xu [STAR Collaboration], Nucl. Phys. A 698 (2002) 607 arXiv:nucl-ex/0104001. For the latest results, see these proceedings.

[5] R. Rapp, G. Chanfray and J. Wambach, Nucl. Phys. A617 (1997) 472;

R. Rapp and J. Wambach, Adv. Nucl. Phys. 25 (2000) 1.

[6] M. F. Lutz and C. L. Korpa, arXiv:nucl-th/0105067

[7] M. Bleicher et al., J. Phys. G25 1859 (1999);

S. A. Bass et al., Prog. Part. Nucl. Phys. 41225 (1998).

[8] M. Bleicher and J. Aichelin, Phys. Lett. B 530 (2002) 81 arXiv:hep-ph/0201123.

[9] M. Bleicher, arXiv:hep-ph/0212378, To appear in Nucl. Phys. A.

[10] J. Rafelski, J. Letessier and G. Torrieri, Phys. Rev. C 64 (2001) 054907 [Erratum-ibid. C 65 (2002) 069902] arXiv:nucl-th/0104042.

[11] G. Torrieri and J. Rafelski, J. Phys. G 28 (2002) 1911 arXiv:hep-ph/0112195.

[12] G. Torrieri and J. Rafelski, arXiv:nucl-th/0212091.

[13] P. Fachini [STAR Collaboration], these proceedings.

[14] E. V. Shuryak and G. E. Brown, Nucl. Phys. A 717 (2003) 322 arXiv:hep-ph/0211119.

[15] P. F. Kolb and M. Prakash, Phys. Rev. C 67 (2003) 044902 arXiv:nucl-th/0301007. 